\title{
An Efficient and Facile Synthesis of 1,2,4-Aryl Triazoles and 4-Thiazolidinones Bearing 6-Fluorochroman Nucleus
}

\author{
Piyush B. Vekariya, Jalpa R. Pandya, and Hitendra S. Joshi \\ Chemical Research Laboratory, Department of Chemistry, Saurashtra University, Rajkot 360005, India \\ Correspondence should be addressed to Hitendra S. Joshi; drhsjoshi49@gmail.com
}

Received 14 April 2014; Accepted 5 July 2014; Published 30 October 2014

Academic Editor: Xin Wang

Copyright (C) 2014 Piyush B. Vekariya et al. This is an open access article distributed under the Creative Commons Attribution License, which permits unrestricted use, distribution, and reproduction in any medium, provided the original work is properly cited.

\begin{abstract}
A new generation of chroman bearing heterocyclic five membered ring such as 1,2,4-triazoles and thiazolidinones was designed and synthesized. New chroman based nucleus 5-(6-fluorochroman-2-yl)-4-aryl-4H-1,2,4-triazole-3-thiol and 6-fluorochroman-N-(4oxo-2-arylthiazolidinin-3-yl) chroman-2-carboxamides were synthesized. Aryl triazole compounds $\mathbf{4 a - 4 j}$ were synthesized from 6-fluorochroman-2-carbohydrazide 2 on reaction with base in methanol and $\mathrm{CS}_{2}$ followed by reaction with substituted aniline. Thiazolidinone compounds $\mathbf{5 a - 5 j}$ were synthesized from 6 -fluorochroman-2-carbohydrazide $\mathbf{2}$ on reaction with substituted aryl aldehyde and thioglycolic acid.
\end{abstract}

\section{Introduction}

The design and synthesis of hybrid molecules encompassing two pharmacophores in one molecular scaffold is a wellestablished approach to the synthesis of more potent drugs with dual activity. With this aspect, 6-fluorochroman-2carboxylic acid derivatives in connection with thiazolidinone and 1, 2, 4-triazoles were found as a promising target for the current research project. 1, 2, 4-Triazoles and 4thiazolidinones are the broadly investigated molecules. They have proved to be the most useful framework for biological activities among nitrogen containing five membered heterocycles. Amongst the diverse classes of heterocyclic compounds chroman, a class of oxygen containing heterocycle forms an important part of many pharmacologically active compounds. For example, the chroman ring is a constituent of various bioactive compounds that are sodium channel blocker [1], $5 \mathrm{HT}_{1 \mathrm{~A}}$ inhibitor [2], and so forth. Commercially available antihypertensive drugs of chroman repinotan [3], robalzotan [4], and specifically 6-fluorochroman nebivolol [5] are well known. Hence, the synthesis of 6-fluorochroman derivatives is currently of significant interest in organic synthesis.
Aryl triazoles comprise various heterocyclic compounds possessing promising biological activity and are found as potential antimicrobial $[6,7]$ and adenosine $A_{2 \mathrm{~A}}$ receptor antagonist [8]. According to the green chemistry approach there are many solvent free reactions of 1, 2, 4-aryl triazoles that have been reported $[9,10]$.

4-Thiazolidinones have been widely explored for their applications in the field of medicine and agriculture [11]. They are also known as promising antimicrobial [12], antiinflammatory [13, 14], antimalerial [15], anticancer [16], tuberculostatic [17], and antiviral agents [18]. Several onepot multicomponent syntheses of 4 -thiazolidinone have been reported [19-21].

\section{Result and Discussion}

The syntheses of triazole and thiazolidinone derivatives have been previously reported by many researchers, and they normally required additional additives and long reaction time. So in this paper, we described an efficient and safe procedure for the synthesis of 4-aryl triazole containing chroman nucleus, using 6-fluorochroman-2-carboxylic acid. 6-Fluorochroman-2-carboxylic acid on esterification with 
<smiles>COC(=O)C1CCc2cc(F)ccc2O1</smiles>

1<smiles>NNC(=O)C1CCc2cc(F)ccc2O1</smiles>

2

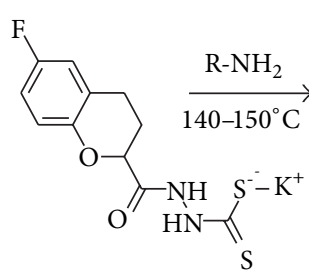

S<smiles>[R]n1c(S)nnc1C1CCc2cc(F)ccc2O1</smiles>

$4 a-4$

3

Scheme 1: Reaction scheme for the synthesis of $\mathbf{4 a - 4 j}$.<smiles>COC(=O)C1CCc2cc(F)ccc2O1</smiles>

1

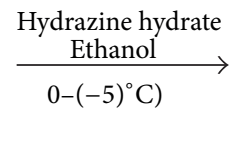

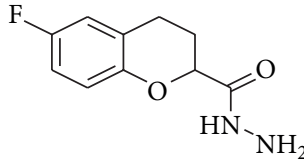

2

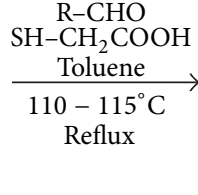<smiles>[R]C1SCC(=O)N1NC(=O)C1CCc2cc(F)ccc2O1</smiles>

$\mathbf{5 a}-\mathbf{5 j}$

$\mathrm{R}=$ aryl

SCHEME 2: Reaction scheme for the synthesis of $\mathbf{5 a}-\mathbf{5 j}$.

methanol in the presence of concentrated $\mathrm{H}_{2} \mathrm{SO}_{4}$ at room temperature gave compound $\mathbf{1}$ (Scheme 1) with good yield which on reaction with hydrazine hydrate (99\%) gave compound 2 . Compound 2 on reaction with carbon disulphide in the presence of $\mathrm{KOH}$ in methanol at RT afforded compound 3. This on further reaction with substituted aniline without use of any solvent in fused condition yielded compounds 4a-4j (Scheme 1). ${ }^{1} \mathrm{H}$ and ${ }^{13} \mathrm{C}$ NMR spectra of the products clearly indicated the formation of triazoles $\mathbf{4 a - 4 j}$ in $75-$ 95\% yields (Table 1). The formation of thiol group - $\mathrm{SH}$ was identified by a sharp singlet at around $\delta=11.43 \mathrm{ppm}$. By the ${ }^{13} \mathrm{C}$ NMR spectrum also supported the presence of $-\mathrm{SH}$ group from the deshielding value of carbon attached to $-\mathrm{SH}$ group at $\delta 168.71 \mathrm{ppm}$.

Here we introduce the one-pot synthesis of thiazolidinone from hydrazide (Scheme 2) with thioglycolic acid, substituted aryl aldehydes in toluene using a Dean-Stark assembly to synthesized compounds $5 \mathbf{a}-5 \mathbf{j}$ (Scheme 2 ). ${ }^{1} \mathrm{H}$ and ${ }^{13} \mathrm{C}$ NMR spectra of the products clearly indicated the formation of 4 -thiazolidinone $\mathbf{5 a - 5 j}$ in $71-95 \%$ yields (Table 2). The formation of $-\mathrm{NH}$ group was identified by a sharp singlet at around $\delta 10.46-10.43 \mathrm{ppm}$, which is further supported by $\mathrm{D}_{2} \mathrm{O}$ exchange. ${ }^{13} \mathrm{C}$ NMR spectrum also supported the presence of amide group from the deshielding value of carbon attached to -CONH group at $\delta 169.3 \mathrm{ppm}$ and carbonyl group (part of a five member ring) at $\delta 169.47 \mathrm{ppm}$.

\section{Conclusion}

In summary, an efficient protocol for the synthesis of new 1 , 2, 4-aryl triazoles and 4-thiazolidinones has been described. Herein, we are reporting the solvent free protocol for the synthesis of $\mathrm{N}$-substituted 1, 2, 4-aryl triazoles (4a-4j) from potassium salt (3). In literature, the synthesis of 4 thiazolidinones was carried out via 2 steps, but, to avoid
TAble 1: Physical data for $\mathbf{4 a - 4 j}$.

\begin{tabular}{lcc}
\hline Entry & Substitution R & Yield (\%) \\
\hline $\mathbf{4 a}$ & 4-CH phenyl & 89 \\
$\mathbf{4 b}$ & 3-Cl phenyl & 86 \\
$\mathbf{4 c}$ & 4-F phenyl & 78 \\
$\mathbf{4 d}$ & 2,5-Dimethyl phenyl & 95 \\
$\mathbf{4 e}$ & 3,4-Dimethyl phenyl & 84 \\
$\mathbf{4 f}$ & 2-F phenyl & 79 \\
$\mathbf{4 g}$ & 2-Cl phenyl & 76 \\
$\mathbf{4 h}$ & 3-Cl-4-F phenyl & 87 \\
$\mathbf{4 i}$ & 2-OCH phenyl & 90 \\
$\mathbf{4 j}$ & 2,5-Difluoro phenyl & 75 \\
\hline
\end{tabular}

multisteps and to make it more viable, we have developed a single-step reaction for the synthesis of 4-thiazolidinones (5a-5j) from hydrazide (2).

\section{Experimental}

Melting points were determined in open capillary tubes and are uncorrected. Formation of the compounds was checked by TLC on silica gel-G plates of $0.5 \mathrm{~mm}$ thickness. IR spectra were recorded on Shimadzu FT-IR-8400 instrument using DRS (diffusive reflectance system) method. Mass spectra were recorded on Shimadzu GC-MS system (model QP-2010) using direct inlet probe technique. ${ }^{1} \mathrm{H}$ NMR and ${ }^{13} \mathrm{C}$ NMR were determined in $\mathrm{CDCl}_{3}$ and $\mathrm{DMSO}-\mathrm{d}_{6}$ on a Bruker $\mathrm{AC}$ $400 \mathrm{MHz}$ and $100 \mathrm{MHz}$ spectrometer. Elemental analysis of all the synthesized compounds was carried out on Euro EA 3000 elemental analyzer and the results are in agreement with the structures assigned. 
TABle 2: Physical data for $\mathbf{5 a} \mathbf{a} \mathbf{5}$.

\begin{tabular}{lcc}
\hline Entry & Substitution $\mathrm{R}$ & Yield (\%) \\
\hline $\mathbf{5 a}$ & 4-OCH & phenyl \\
$\mathbf{5 b}$ & 2- $\mathrm{NO}_{2}$ phenyl & 85 \\
$\mathbf{5 c}$ & 4-F phenyl & 95 \\
$\mathbf{5 d}$ & 3-Cl phenyl & 89 \\
$\mathbf{5 e}$ & 4-Br phenyl & 77 \\
$\mathbf{5 f}$ & 4-OH phenyl & 90 \\
$\mathbf{5 g}$ & 4- $\mathrm{CH}_{3}$ phenyl & 73 \\
$\mathbf{5 h}$ & 2-Cl phenyl & 84 \\
$\mathbf{5 i}$ & 4- $\mathrm{NO}_{2}$ phenyl & 71 \\
$\mathbf{5 j}$ & 3-Br phenyl & 79 \\
\hline
\end{tabular}

4.1. General Procedure for the Synthesis of Functionalized 1, 2, 4-Aryl Triazoles $(\mathbf{4 a - 4 j )}$. To a stirred solution of 6fluorochroman-2-carboxylic acid $(0.01 \mathrm{~mol})$ in methanol at room temperature, concentrated $\mathrm{H}_{2} \mathrm{SO}_{4}(0.01 \mathrm{~mol})$ was added and reaction mixture was allowed to stir at RT for 10 hours. After completion of the reaction, solvent was evaporated and the resulting mass was poured on to ice, neutralized with saturated sodium bicarbonate solution. Separated solid precipitate was filtered, washed with water, and dried to afford methyl 6-fluorochroman-2-carboxylate 1.

Compound $1(0.01 \mathrm{~mol})$ in absolute ethanol was taken into the RBF and cooled at $(-5)^{\circ} \mathrm{C}$. To the previously cooled solution hydrazine hydrate $(99 \%, 0.08 \mathrm{~mol})$ was added and reaction mixture was allowed to stir at $0-(-5)^{\circ} \mathrm{C}$ for 10 hours. After the completion of reaction separated solid residues were filtered, washed with cold ethanol, and dried to afford 6fluorochroman-2-carbohydrazide 2, yield: $2.0 \mathrm{~g}$ (98\%).

To a mixture of compound $2(0.1 \mathrm{~mol})$ and potassium hydroxide $(0.15 \mathrm{~mol})$ in methanol carbon disulphide $(0.15 \mathrm{~mol})$ was added dropwise. Reaction mass was allowed to stir at RT for 22-24 hours. After completion of reaction the obtained solid was filtered, washed with diethyl ether, and dried to afford compound 3 . There is no need to purify the salt for further reaction.

An equimolar mixture of potassium 2-[(6-fluorochroman-2-yl) carbonyl] hydrazine carbodithioate $3(0.01 \mathrm{~mol})$ and substituted aniline $(0.01 \mathrm{~mol})$ was taken in RBF and heated at $140-150^{\circ} \mathrm{C}$ for $12-15$ hours until the evolution of $\mathrm{H}_{2} \mathrm{~S}$ gas ceased. After completion of reaction solid residue was dissolved in DMF, treated with dilute $\mathrm{HCl}$, and poured on crushed ice. The product was isolated and crystallized from ethanol to give compounds $\mathbf{4 a - 4 j}$ as analytical pure product.

4.1.1. 5-(6-Fluorochroman-2-yl)-4-(p-tolyl)-4H-1, 2, 4-triazole3-thiol (4a). Yield 89\%, mp 170-172 ${ }^{\circ}$; IR (DRS): 3076(Ar, C-H str.), 2924(C-H str.), 2573(-SH str.), 1735(C=O str.), 1087(C-N str.), 1041 (C-O-C str.) $\mathrm{cm}^{-1}$; ${ }^{1} \mathrm{H}$ NMR $(400 \mathrm{MHz}$, $\mathrm{CDCl}_{3}$ ): $\delta \mathrm{ppm} 2.02-2.44(\mathrm{~m}, 5 \mathrm{H}, 2 \mathrm{CH}, 3 \mathrm{CH}), 2.65-2.96(\mathrm{~m}$, $2 \mathrm{H}, 2 \mathrm{CH}), 4.83-4.90(\mathrm{dd}, J=12.6 \mathrm{~Hz}, 6 \mathrm{~Hz}, 1 \mathrm{H}, \mathrm{CH}), 6.60-$ 6.64(m, $1 \mathrm{H}, \mathrm{ArH}), 6.73-6.76(\mathrm{~m}, 2 \mathrm{H}, \mathrm{ArH}), 7.05-7.49(\mathrm{~m}$, $4 \mathrm{H}, \mathrm{ArH}), 11.43(\mathrm{~s}, 1 \mathrm{H}, \mathrm{SH}) .{ }^{13} \mathrm{C}$ NMR $\left(100 \mathrm{MHz}, \mathrm{CDCl}_{3}\right)$ : $\delta$ ppm $\quad 20.3\left(\mathrm{CH}_{3}\right), \quad 26.27\left(\mathrm{CH}_{2}\right), \quad 28.87\left(\mathrm{CH}_{2}\right), \quad 85.3(\mathrm{CH})$, $108.52(\mathrm{CH}), 112.98(\mathrm{CH}), 116.81(\mathrm{CH}), 119.54(\mathrm{CH}), 129.2(\mathrm{C})$,
131.16(C), 133.09(CH), 136.12(C), 139.69(C), $155.8(\mathrm{C})$, 158.29(C), 173.99(C). MS: $m / z=341[\mathrm{M}]^{+}$; Anal. Calcd for $\mathrm{C}_{18} \mathrm{H}_{16} \mathrm{FN}_{3} \mathrm{OS}$ : C, 63.32; H, 4.72; N, 12.31. Found: C, 63.23; $\mathrm{H}, 4.41 ; \mathrm{N}, 12.28 \%$.

4.1.2. 4-(3-Chlorophenyl)-5-(6-fluorochroman-2-yl)-4H-1, 2, 4-triazole-3-thiol (4b). Yield $86 \%, \mathrm{mp} 205-207^{\circ} \mathrm{C}$; IR (DRS): 3066(Ar, C-H str.), 2914(C-H str.), 2850(C-H str.), 2533(-SH str.), 1558(Ar, $\mathrm{C}=\mathrm{C}$ bend.), 1375(C-H ben), $800(\mathrm{C}-\mathrm{Cl}$ str.), 1087(C-N str.), 1041 (C-O-C str.) cm ${ }^{-1} ;{ }^{1} \mathrm{H}$ NMR $(400 \mathrm{MHz}$, DMSO $\left.-\mathrm{d}_{6}\right): \delta \mathrm{ppm} 2.27-2.30(\mathrm{~m}, 2 \mathrm{H}, 2 \mathrm{CH}), 2.82-2.95(\mathrm{~m}$, $2 \mathrm{H}, 2 \mathrm{CH}), 4.92-4.95(\mathrm{dd}, J=6.12 \mathrm{~Hz}, 12 \mathrm{~Hz}, 1 \mathrm{H}, \mathrm{CH})$, 6.50-6.54(m, 1H, ArH), 6.75-6.79(m, 2H, ArH), 7.41-7.43(m, $1 \mathrm{H}, \mathrm{ArH}), 7.53-7.55(\mathrm{~m}, 3 \mathrm{H}, \mathrm{ArH}), 14.0(\mathrm{~s}, 1 \mathrm{H}, \mathrm{SH}) .{ }^{13} \mathrm{C} \mathrm{NMR}$ $\left(100 \mathrm{MHz}\right.$, DMSO-d $\left.{ }_{6}\right): \delta \mathrm{ppm} \quad 25.27\left(\mathrm{CH}_{2}\right), \quad 28.27\left(\mathrm{CH}_{2}\right)$, 85.3(CH), $112.98(\mathrm{CH}), 116.81(\mathrm{CH}), 120.08(\mathrm{CH}), 122.3(\mathrm{CH})$, $125.68(\mathrm{CH}), \quad 129.2(\mathrm{C}), 133.41(\mathrm{CH}), 138.88(\mathrm{C}), 139.69(\mathrm{C})$, 142.37(C), 155.8(C), 158.29(C), 173.99(C) MS: $m / z=$ $361[\mathrm{M}]^{+}$; Anal. Calcd for $\mathrm{C}_{17} \mathrm{H}_{13} \mathrm{ClFN}_{3}$ OS: C, 56.43; H, 3.62; $\mathrm{N}, 11.61$. Found: C, $56.18 ; \mathrm{H}, 3.49 ; \mathrm{N}, 11.59 \%$.

4.1.3. 5-(6-Fluorochroman-2-yl)-4-(4-fluorophenyl)-4H-1,2,4triazole-3-thiol (4c). Yield $78 \%$, mp $188-190^{\circ} \mathrm{C}$; IR (DRS): 3030(Ar, C-H str.), 2558(-SH str.), 1581(-C=C-, str.), 1225 (C-O-C str.) $\mathrm{cm}^{-1}$; ${ }^{1} \mathrm{H}$ NMR $\left(400 \mathrm{MHz}, \mathrm{CDCl}_{3}\right)$ : $\delta \mathrm{ppm} 2.21-2.46(\mathrm{~m}, 2 \mathrm{H}, 2 \mathrm{CH}), 2.75-2.85(\mathrm{~m}, 2 \mathrm{H}, 2 \mathrm{CH})$, $4.88-4.93(\mathrm{dd}, J=12.6 \mathrm{~Hz}, 6 \mathrm{~Hz}, 1 \mathrm{H}, \mathrm{CH}), 6.51-6.55(\mathrm{~m}$, $1 \mathrm{H}, \mathrm{ArH}), 6.77-6.81(\mathrm{~m}, 2 \mathrm{H}, \mathrm{ArH}), 7.53-7.57(\mathrm{~m}, 3 \mathrm{H}, \mathrm{ArH})$, $13.05(\mathrm{~s}, 1 \mathrm{H}, \mathrm{SH}) .{ }^{13} \mathrm{C}$ NMR $\left(100 \mathrm{MHz}, \mathrm{CDCl}_{3}\right): 26.27\left(\mathrm{CH}_{2}\right)$, 28.87 $\left(\mathrm{CH}_{2}\right), 85.3(\mathrm{CH}), 108.52(\mathrm{CH}), 112.98(\mathrm{CH}), 116.81(\mathrm{CH})$, $118.6(\mathrm{CH}), \quad 122.97(\mathrm{CH}), \quad 129.2(\mathrm{C}), \quad 133.7(\mathrm{C}), \quad 139.69(\mathrm{C})$, 155.8(C), 158.29(C), 160.51(C), 173.99(C). MS: $m / z=345$ $[\mathrm{M}]^{+}$; Anal. Calcd for $\mathrm{C}_{17} \mathrm{H}_{13} \mathrm{~F}_{2} \mathrm{~N}_{3}$ OS: C, 59.12; $\mathrm{H}, 3.79 ; \mathrm{N}$, 12.17. Found: C, 59.02; H, 3.53; N, $12.01 \%$.

4.1.4. 4-(2, 5-Dimethylphenyl)-5-(6-fluorochroman-2-yl)-4H1, 2, 4-triazole-3-thiol (4d). Yield 95\%, mp $123-125^{\circ} \mathrm{C}$; IR (DRS): 3074(Ar, C-H str.), 2984(C-H str.), 1645(-C=C-, str.), $1468\left(\mathrm{C}-\mathrm{H}\right.$ bending $\mathrm{cm}^{-1} ;{ }^{1} \mathrm{H}$ NMR $\left(400 \mathrm{MHz}, \mathrm{CDCl}_{3}\right)$ : $\delta \mathrm{ppm} 1.92(\mathrm{~s}, 6 \mathrm{H}, 3 \mathrm{CH}, 3 \mathrm{CH}), 2.21-2.46(\mathrm{~m}, 2 \mathrm{H}, 2 \mathrm{CH})$, $2.75-2.85(\mathrm{~m}, 2 \mathrm{H}, 2 \mathrm{CH}), 4.85-4.92(\mathrm{dd}, J=12.6 \mathrm{~Hz}$, $6 \mathrm{~Hz}, 1 \mathrm{H}, \mathrm{CH}), 6.72-6.95(\mathrm{~m}, 3 \mathrm{H}, \mathrm{ArH}), 7.29-7.43(\mathrm{~m}, 3 \mathrm{H}$, $\mathrm{ArH}), 13.05(\mathrm{~s}, 1 \mathrm{H}, \mathrm{SH}) .{ }^{13} \mathrm{C}$ NMR $\left(100 \mathrm{MHz}, \mathrm{CDCl}_{3}\right)$ : $\delta \mathrm{ppm} \quad 17.98\left(\mathrm{CH}_{3}\right), \quad 26.87\left(\mathrm{CH}_{2}\right), \quad 28.87\left(\mathrm{CH}_{2}\right), \quad 30.2\left(\mathrm{CH}_{3}\right)$, 85.3(CH), 108.52(CH), $116.81(\mathrm{CH}), 118.6(\mathrm{CH}), 112.98(\mathrm{CH})$, $124.19(\mathrm{CH}), 132.61(\mathrm{CH}), 134.95(\mathrm{C}), 138.13(\mathrm{C}), 140.75(\mathrm{C})$, 146.2(C), 155.8(C), 158.29(C), 175.05(C). MS: $m / z=355[\mathrm{M}]^{+}$; Anal. Calcd for $\mathrm{C}_{19} \mathrm{H}_{18} \mathrm{FN}_{3}$ OS: C, 64.21; H, 5.10; N, 11.82 . Found: C, 64.16; H, 4.93; N, 11.78\%.

4.1.5. 4-(3, 4-Dimethylphenyl)-5-(6-fluorochroman-2-yl)-4H-1, 2, 4-triazole-3-thiol (4e). Yield $84 \%, \mathrm{mp} 163-165^{\circ} \mathrm{C}$; IR (DRS): 3081(Ar, C-H str.), 2975(C-H str.), 2575(-SH str.), 1641(C-H bending), 1579(-C=C-, str.), 1142(C-F str.) $\mathrm{cm}^{-1}$; ${ }^{1} \mathrm{H}$ NMR $\left(400 \mathrm{MHz}, \mathrm{CDCl}_{3}\right): \delta \mathrm{ppm} 2.34(\mathrm{~s}, 6 \mathrm{H}, 3 \mathrm{CH}, 3 \mathrm{CH})$, $2.21-2.46(\mathrm{~m}, 2 \mathrm{H}, 2 \mathrm{CH}), 2.75-2.85(\mathrm{~m}, 2 \mathrm{H}, 2 \mathrm{CH}), 4.85-$ $4.92(\mathrm{dd}, J=12.6 \mathrm{~Hz}, 6 \mathrm{~Hz}, 1 \mathrm{H}, \mathrm{CH}), 6.72-6.95(\mathrm{~m}, 3 \mathrm{H}, \mathrm{ArH})$, $7.29-7.43(\mathrm{~m}, 3 \mathrm{H}, \mathrm{ArH}), 13.05(\mathrm{~s}, 1 \mathrm{H}, \mathrm{SH}) .{ }^{13} \mathrm{C}$ NMR $(100 \mathrm{MHz}$, 
$\left.\mathrm{CDCl}_{3}\right): \quad \delta \mathrm{ppm} \quad 19.0\left(\mathrm{CH}_{3}\right), \quad 20.01\left(\mathrm{CH}_{3}\right), \quad 26.27\left(\mathrm{CH}_{2}\right)$, 28.87 $\left(\mathrm{CH}_{2}\right), 85.3(\mathrm{CH}), 112.98(\mathrm{CH}), 116.81(\mathrm{CH}), 117.91(\mathrm{CH})$, 124.04(CH), 129.2(C), 132.85(C), 133.52(C), 139.69(C), 141.78(C), 143.79(C), 155.8(C), 158.29(C), 173.998(C). MS: $m / z=355[\mathrm{M}]^{+}$; Anal. Calcd for $\mathrm{C}_{19} \mathrm{H}_{18} \mathrm{FN}_{3} \mathrm{OS}$ : C, 64.21; $\mathrm{H}$, 5.10; N, 11.82. Found: C, 64.09; H, 5.03; N, 11.50\%.

4.1.6. 5-(6-Fluorochroman-2-yl)-4-(2-fluorophenyl)-4H-1,2, 4triazole-3-thiol (4f). Yield 79\%, mp 108-110 C; IR (DRS): 3080(Ar, C-H str.), 2983(C-H str.), 2561(-SH str.), 1629(C-H bending), 1572(C-H bending), 1525( $-\mathrm{C}=\mathrm{C}-$, str.) $1196\left(\mathrm{C}-\mathrm{O}-\mathrm{C}\right.$ str. $\mathrm{cm}^{-1} ;{ }^{1} \mathrm{H}$ NMR $\left(400 \mathrm{MHz}, \mathrm{CDCl}_{3}\right)$ : $\delta \mathrm{ppm} 2.34(\mathrm{~s}, 6 \mathrm{H}, 3 \mathrm{CH}, 3 \mathrm{CH}), 2.21-2.46(\mathrm{~m}, 2 \mathrm{H}, 2 \mathrm{CH})$, 2.75-2.85(m, 2H, 2CH), 4.90-4.97(dd, $J=12.6 \mathrm{~Hz}, 6 \mathrm{~Hz}$, $1 \mathrm{H}, \mathrm{CH}), 6.72-6.95(\mathrm{~m}, 3 \mathrm{H}, \mathrm{ArH}), 7.24-7.75(\mathrm{~m}, 3 \mathrm{H}, \mathrm{ArH})$, 13.05(s, $1 \mathrm{H}, \mathrm{SH}) .{ }^{13} \mathrm{C} \mathrm{NMR}\left(100 \mathrm{MHz}, \mathrm{CDCl}_{3}\right): \delta \mathrm{ppm}$ 26.27 $\left(\mathrm{CH}_{2}\right), 28.87\left(\mathrm{CH}_{2}\right), 85.3(\mathrm{CH}), 108.52(\mathrm{CH}), 112.98(\mathrm{CH})$, $116.81(\mathrm{CH}), \quad 119.7(\mathrm{C}), \quad 119.58(\mathrm{CH}), \quad 129.2(\mathrm{C}), \quad 129.95(\mathrm{CH})$, $130.09(\mathrm{CH}), 130.97(\mathrm{CH}), 139.69(\mathrm{C}), 155.8(\mathrm{C}), 158.29(\mathrm{C})$, 162.56(C), 173.99(C). MS: $m / z=345[\mathrm{M}]^{+}$; Anal. Calcd for $\mathrm{C}_{17} \mathrm{H}_{13} \mathrm{~F}_{2} \mathrm{~N}_{3}$ OS: C, 59.12; H, 3.79; N, 12.17. Found: C, 58.96; $\mathrm{H}, 3.67 ; \mathrm{N}, 12.06 \%$.

4.1.7. 4-(2-Chlorophenyl)-5-(6-fluorochroman-2-yl)-4H-1, 2, 4triazole-3-thiol (4g). Yield 76\%, mp 192-194 C; IR (DRS): 3077(Ar, C-H str.), 2978(C-H str.), 2563(-SH str.), 1563(-C=C str.), 1464(H-C-H bend), 1310(C-O str.), $870\left(\mathrm{C}-\mathrm{Cl}\right.$ str. $\mathrm{cm}^{-1} ;{ }^{1} \mathrm{H}$ NMR $\left(400 \mathrm{MHz}, \mathrm{CDCl}_{3}\right): \delta \mathrm{ppm}$ $2.34(\mathrm{~s}, 6 \mathrm{H}, 3 \mathrm{CH}, 3 \mathrm{CH}), 2.21-2.46(\mathrm{~m}, 2 \mathrm{H}, 2 \mathrm{CH}), 2.75-2.85(\mathrm{~m}$, $2 \mathrm{H}, 2 \mathrm{CH}), 4.90-4.97(\mathrm{dd}, J=12.6 \mathrm{~Hz}, 6 \mathrm{~Hz}, 1 \mathrm{H}, \mathrm{CH})$, 6.72-6.95(m, 3H, ArH), 7.39-7.50(m, 3H, ArH), 13.05(s, $1 \mathrm{H}, \mathrm{SH}) .{ }^{13} \mathrm{C} \mathrm{NMR}\left(100 \mathrm{MHz}, \mathrm{CDCl}_{3}\right): \delta \mathrm{ppm} \mathrm{26.27}\left(\mathrm{CH}_{2}\right)$, 28.87 $\left(\mathrm{CH}_{2}\right), 85.3(\mathrm{CH}), 108.52(\mathrm{CH}), 112.98(\mathrm{CH}), 116.81(\mathrm{CH})$, $127.83(\mathrm{CH}), 126.39(\mathrm{C}), 128.04(\mathrm{CH}), 129.2(\mathrm{C}), 131.85(\mathrm{CH})$, 132.57(CH), 140.8(C), 142.53(C), 155.8(C), 158.29(C), 175.1(C). MS: $m / z=361[\mathrm{M}]^{+}$; Anal. Calcd $\mathrm{C}_{17} \mathrm{H}_{13} \mathrm{ClFN}_{3} \mathrm{OS}$ : C, 56.43; H, 3.62; N, 11.61. Found: C, 55.97; H, 3.55; N, 11.59\%.

4.1.8. 4-(3-Chloro-4-fluorophenyl)-5-(6-fluorochroman-2-yl)4H-1,2,4-triazole-3-thiol (4h). Yield 87\%, mp 139-141 ${ }^{\circ} \mathrm{C}$; IR (DRS): 3075(Ar, C-H str.), 2553(-SH str.), 1581(-C=C-, str.), 1423(H-C-H bend), 1281 (C-O str.), $870\left(\mathrm{C}-\mathrm{Cl}\right.$ str. $\mathrm{cm}^{-1}$; ${ }^{1} \mathrm{H}$ NMR $\left(400 \mathrm{MHz}, \mathrm{DMSO}-\mathrm{d}_{6}\right): \delta \mathrm{ppm} 2.34(\mathrm{~s}, 6 \mathrm{H}, 3 \mathrm{CH}$, $3 \mathrm{CH}), 2.21-2.46(\mathrm{~m}, 2 \mathrm{H}, 2 \mathrm{CH}), 2.75-2.85(\mathrm{~m}, 2 \mathrm{H}, 2 \mathrm{CH})$, $4.90-4.97(\mathrm{dd}, J=12.6 \mathrm{~Hz}, 6 \mathrm{~Hz}, 1 \mathrm{H}, \mathrm{CH}), 6.72-6.95(\mathrm{~m}, 3 \mathrm{H}$, ArH), 7.18-7.79(m, 3H, ArH), 13.05(s, 1H, SH). ${ }^{13} \mathrm{C}$ NMR $\left(100 \mathrm{MHz}, \quad \mathrm{DMSO}-\mathrm{d}_{6}\right): \delta \mathrm{ppm} \quad 26.27\left(\mathrm{CH}_{2}\right), \quad 28.87\left(\mathrm{CH}_{2}\right)$, 85.3(CH), 108.52(CH), $112.98(\mathrm{CH}), 116.81(\mathrm{CH}), 120.08(\mathrm{CH})$, 121.91(CH), 124.85(C), 125.71(CH), 129.2(C), 139.69(C), 141.09(C), 155.8(C), 156.41(C), 158.29(C), 173.99(C). MS: $m / z$ $=379[\mathrm{M}]^{+}$; Anal. Calcd for $\mathrm{C}_{17} \mathrm{H}_{12} \mathrm{ClF}_{2} \mathrm{~N}_{3} \mathrm{OS}$ : C, 53.76; $\mathrm{H}$, 3.18; N, 11.06. Found: C, 53.69; H, 3.07; N, 10.90\%.

4.1.9. 5-(6-Fluorochroman-2-yl)-4-(2-methoxyphenyl)-4H-1, 2, 4-triazole-3-thiol (4i). Yield 90\%, mp 251-253 ${ }^{\circ} \mathrm{C}$; IR (DRS): 3075(Ar, C-H str.), 2553(-SH str.), 1581(-C=C-, str.), $1423(\mathrm{H}-\mathrm{C}-\mathrm{H}$ bend), 1281 (C-O str.), $870(\mathrm{C}-\mathrm{Cl}$ str.), 1080(C-F str.) $\mathrm{cm}^{-1} ;{ }^{1} \mathrm{H}$ NMR (400 MHz, DMSO-d 6 ): $\delta \mathrm{ppm}$ $2.34(\mathrm{~s}, 6 \mathrm{H}, 3 \mathrm{CH}, 3 \mathrm{CH}), 2.21-2.46(\mathrm{~m}, 2 \mathrm{H}, 2 \mathrm{CH}), 2.75-2.85(\mathrm{~m}$, $2 \mathrm{H}, 2 \mathrm{CH}), 3.83(\mathrm{~s}, 3 \mathrm{H}, \mathrm{OCH} 3), 4.90-4.97(\mathrm{dd}, J=12.6 \mathrm{~Hz}$, $6 \mathrm{~Hz}, 1 \mathrm{H}, \mathrm{CH}), 6.72-6.95(\mathrm{~m}, 3 \mathrm{H}, \mathrm{ArH}), 6.99-7.51(\mathrm{~m}, 4 \mathrm{H}$, $\mathrm{ArH}), 13.05(\mathrm{~s}, 1 \mathrm{H}, \mathrm{SH}) .{ }^{13} \mathrm{C} \mathrm{NMR}\left(100 \mathrm{MHz}, \mathrm{DMSO}-\mathrm{d}_{6}\right)$ : $\delta$ ppm $26.27\left(\mathrm{CH}_{2}\right), \quad 28.87\left(\mathrm{CH}_{2}\right), \quad 56.32\left(\mathrm{CH}_{3}\right), \quad 85.3(\mathrm{CH})$, $108.52(\mathrm{CH}), 112.98(\mathrm{CH}), 115.51(\mathrm{CH}), 116.81(\mathrm{CH}), 120.83(\mathrm{CH})$, 123.69(CH), 129.2(C), 131.19(CH), 134.73(C), 139.24(C), 155.8(C), 157.76(C), 158.29(C), 173.54(C). MS: $m / z=357$ $[\mathrm{M}]^{+}$; Anal. Calcd for $\mathrm{C}_{18} \mathrm{H}_{16} \mathrm{FN}_{3} \mathrm{O}_{2} \mathrm{~S}: \mathrm{C}, 60.49 ; \mathrm{H}, 4.51 ; \mathrm{N}$, 11.76. Found: C, 60.39; H, 4.29; N, 11.37\%.

4.1.10. 4-(2, 5-Difluorophenyl)-5-(6-fluorochroman-2-yl)-4H1,2,4-triazole-3-thiol (4j). Yield 75\%, mp 229-231 ${ }^{\circ} \mathrm{C}$; IR (DRS): 3061(Ar, C-H str.), 2951 (C-H str.), 2535(-SH str.), 1589(-C=C-, str.), 1462(H-C-H bend), 1520(C-O str.), 1075 (C-F str.) $\mathrm{cm}^{-1} ;{ }^{1} \mathrm{H}$ NMR $\left(400 \mathrm{MHz}, \mathrm{CDCl}_{3}\right): \delta \mathrm{ppm}$ $2.34(\mathrm{~s}, 6 \mathrm{H}, 3 \mathrm{CH}, 3 \mathrm{CH}), 2.21-2.46(\mathrm{~m}, 2 \mathrm{H}, 2 \mathrm{CH}), 2.75-$ 2.85(m, 2H, 2CH), 3.83(s, 3H, OCH3), 4.90-4.97(dd, $J=$ $12.6 \mathrm{~Hz}, 6 \mathrm{~Hz}, 1 \mathrm{H}, \mathrm{CH}), 6.72-6.95(\mathrm{~m}, 3 \mathrm{H}, \mathrm{ArH}), 7.13-7.22(\mathrm{~m}$, $3 \mathrm{H}, \mathrm{ArH}), 13.05(\mathrm{~s}, 1 \mathrm{H}, \mathrm{SH}) .{ }^{13} \mathrm{C} \mathrm{NMR}\left(100 \mathrm{MHz}, \mathrm{CDCl}_{3}\right)$ : 26.27(CH2), 28.87(CH2), 85.3(CH), 108.29(CH), $108.69(\mathrm{CH})$ $112.98(\mathrm{CH}), 116.81(\mathrm{CH}), 117.32(\mathrm{CH}), 118.79(\mathrm{C}), 127.11(\mathrm{CH})$, 129.2(C), 139.69(C), 155.8(C), 158.29(C), 159.93(C), 173.54(C). MS: $m / z=363[\mathrm{M}]^{+}$; Anal. Calcd for $\mathrm{C}_{17} \mathrm{H}_{12} \mathrm{~F}_{3} \mathrm{~N}_{3} \mathrm{O}_{2} \mathrm{~S}: \mathrm{C}$, 56.19; H, 3.33; N, 11.56. Found: C, 56.06; H, 3.14; N, 11.32\%.

4.2. General Procedure for the Synthesis of Functionalized 4-Thiazolidinones $5 \boldsymbol{a}-\mathbf{5 j}$. An equimolar mixture of 6fluorochroman-2-carbohydrazide $2(0.01 \mathrm{~mol})$ and different aryl aldehydes $(0.01 \mathrm{~mol})$ was taken in $\mathrm{RBF}$ and to this, thioglycolic acid (mercaptoacetic acid) $(0.29 \mathrm{~mol})$ in toluene was added. Then reaction mixture was allowed to reflux in a Dean-Stark assembly with continuous stirring. After completion of the reaction ( 48 hrs monitoring by TLC), the content was cooled to room temperature and then neutralized with saturated sodium bicarbonate solution. The organic extracts were washed with water and dried over $\mathrm{Na}_{2} \mathrm{SO}_{4}$. The solvent was evaporated in vacuo and the resulting crude product was purified by column chromatography to give the analytical pure compounds $\mathbf{5 a - 5 j}$. Column chromatography was carried out in hexane: ethyl acetate solvent system. Pure compound was eluted in $23 \%$ ethyl acetate in hexane.

4.2.1. 6-Fluoro-N-(2-(4-methoxyphenyl)-4-oxothiazolidine-3yl) chroman-2-carboxamide (5a). Yield $85 \%, \mathrm{mp} 98-100^{\circ} \mathrm{C}$; IR (DRS): 3383(Amide-NH str.), 3081(Ar, C-H str.), 2958(C$\mathrm{H}$ str.), $1714(\mathrm{C}=\mathrm{O}$ str.), 1688 (amide $\mathrm{C}=\mathrm{O}$ str.), $1542(-\mathrm{NH}$ bend), 1365 (C-F str.), $1278\left(\mathrm{C}-\mathrm{O}-\mathrm{C}\right.$ str.) $\mathrm{cm}^{-1} ;{ }^{1} \mathrm{H}$ NMR $\left(400 \mathrm{MHz}, \mathrm{DMSO}-\mathrm{d}_{6}\right): \delta \mathrm{ppm} 2.15-2.40(\mathrm{~m}, 2 \mathrm{H}, 2 \mathrm{CH}), 2.75-$ $2.85(\mathrm{~m}, 2 \mathrm{H}, 2 \mathrm{CH}), 3.83(\mathrm{~s}, 3 \mathrm{H}, \mathrm{OCH} 3), 4.69-4.72(\mathrm{~d}, J=$ $16.0 \mathrm{~Hz}, 1 \mathrm{H}, \mathrm{CH}), 3.91-3.95(\mathrm{~d}, J=15.6 \mathrm{~Hz}, 1 \mathrm{H}, \mathrm{CH}), 4.69-$ $4.72(\mathrm{dd}, J=3.6 \mathrm{~Hz}, 3.2 \mathrm{~Hz}, 1 \mathrm{H}, \mathrm{CH}), 5.92(\mathrm{~s}, 1 \mathrm{H}, \mathrm{CH})$, 6.72-6.95(m, 3H, ArH), 6.87-7.84(m, 4H, ArH), 8.0(s, 1H, $\mathrm{NH}),{ }^{13} \mathrm{C}$ NMR $\left(100 \mathrm{MHz}, \mathrm{DMSO}-\mathrm{d}_{6}\right): \delta \mathrm{ppm} 25.25\left(\mathrm{CH}_{2}\right)$, 26.19 $\left(\mathrm{CH}_{2}\right), 34.2\left(\mathrm{CH}_{2}\right), 55.33\left(\mathrm{CH}_{3}\right), 70.50(\mathrm{CH}), 85.58(\mathrm{CH})$, 108.7(CH), 112.44(CH), 114.1(CH), 116.43(CH), 126.91(CH), 129.9(C), 133.68(C), 154.35(C), $157.18(\mathrm{C}), 155.8(\mathrm{C}), 158.08(\mathrm{C})$, 
165.35(C), 169.36(C). MS: $m / z=402[\mathrm{M}]^{+}$; Anal. Calcd for $\mathrm{C}_{20} \mathrm{H}_{19} \mathrm{FN}_{2} \mathrm{O}_{4} \mathrm{~S}$ : C, 59.69; H, 4.76; N, 6.96. Found: C, 59.23; $\mathrm{H}, 4.61 ; \mathrm{N}, 6.88 \%$.

4.2.2. 6-Fluoro-N-(2-(2-nitrophenyl)-4-oxothiazolidine-3-yl) chroman-2-carboxamide (5b). Yield 95\%, mp 190-192 C; IR (DRS): 3392(Amide-NH str.), 3205(Ar, C-H str.), 1712(C=O str.), 1678(amide $\mathrm{C}=\mathrm{O}$ str.), 1523( $-\mathrm{NH}$ bend), 1590( $\mathrm{N}=\mathrm{O}$ str.), 1259 (C-O-C str.), 1190 (C-F str.) $\mathrm{cm}^{-1}$; ${ }^{1} \mathrm{H}$ NMR $(400 \mathrm{MHz}$, DMSO- $\left.\mathrm{d}_{6}\right): \delta$ ppm 1.81-2.05(m, $\left.2 \mathrm{H}, 2 \mathrm{CH}\right), 2.61-2.74(\mathrm{~m}, 2 \mathrm{H}$, $2 \mathrm{CH}), 3.68-3.72(\mathrm{~d}, J=16.0 \mathrm{~Hz}, 1 \mathrm{H}, \mathrm{CH}), 3.91-3.95(\mathrm{~d}, J=$ $15.6 \mathrm{~Hz}, 1 \mathrm{H}, \mathrm{CH}), 4.66-4.69(\mathrm{dd}, J=3.6 \mathrm{~Hz}, 3.2 \mathrm{~Hz}, 1 \mathrm{H}, \mathrm{CH})$, 6.12(s, 1H, CH), 6.72-6.79(m, 1H, ArH), 6.84-6.91(m, 2H, ArH), 7.60-7.63(t, 1H, ArH), 7.78-7.87(m, 2H, ArH), 8.04$8.06(\mathrm{~d}, J=8.0 \mathrm{~Hz}, 1 \mathrm{H}, \mathrm{ArH}), 10.43-10.46(\mathrm{~d}, J=11.6 \mathrm{~Hz}, 1 \mathrm{H}$, $\mathrm{NH}) .{ }^{13} \mathrm{C}$ NMR $\left(100 \mathrm{MHz}, \mathrm{DMSO}-\mathrm{d}_{6}\right): \delta \mathrm{ppm} 25.25\left(\mathrm{CH}_{2}\right)$, 26.19 $\left(\mathrm{CH}_{2}\right), 34.2\left(\mathrm{CH}_{2}\right), 68.3(\mathrm{CH}), 85.58(\mathrm{CH}), 108.7(\mathrm{CH})$, $114.1(\mathrm{CH}), 116.43(\mathrm{CH}), 123.91(\mathrm{CH}), 125.5(\mathrm{C}), 129.87(\mathrm{CH})$, 129.9(C), 127.85(CH), 132.06(C), 149.87(C), 154.35(C), 157.18 (C), 169.36(C). MS: $m / z=417[\mathrm{M}]^{+}$; Anal. Calcd for $\mathrm{C}_{19} \mathrm{H}_{16} \mathrm{FN}_{3} \mathrm{O}_{5} \mathrm{~S}: \mathrm{C}, 54.67 ; \mathrm{H}, 3.86 ; \mathrm{N}, 10.07$. Found: C, 54.58; $\mathrm{H}, 3.49$; N, 9.99\%.

4.2.3. 6-Fluoro-N-(2-(4-fluorophenyl)-4-oxothiazolidine-3-yl) chroman-2-carboxamide (5c). Yield $89 \%, \mathrm{mp} 106-110^{\circ} \mathrm{C}$; IR (DRS): 3487(Amide-NH str.), 3041(Ar, C-H str.), 1710(C=O str.), 1676(amide $\mathrm{C}=\mathrm{O}$ str.), 1537( $-\mathrm{NH}$ bend), 1201(C-O-C str.), 1190 (C-F str.) $\mathrm{cm}^{-1}$; ${ }^{1} \mathrm{H}$ NMR $\left(400 \mathrm{MHz}, \mathrm{CDCl}_{3}\right)$ : $\delta$ ppm 1.82-2.02(m, 2H, 2CH), 2.56-2.70(m, 2H, 2CH), 3.59$3.78(\mathrm{~m}, 2 \mathrm{H}, 2 \mathrm{CH}), 4.45-4.58(\mathrm{dd}, J=10.52 \mathrm{~Hz}, 11.52 \mathrm{~Hz}$, $1 \mathrm{H}, \mathrm{CH}), 5.79-5.83(\mathrm{~d}, J=17.32 \mathrm{~Hz}, 1 \mathrm{H}, \mathrm{CH}), 6.54-6.72(\mathrm{~m}$, $3 \mathrm{H}, \mathrm{ArH}), 6.86-6.90(\mathrm{t}, 1 \mathrm{H}, \mathrm{ArH}), 6.99-7.03(\mathrm{t}, 1 \mathrm{H}, \mathrm{ArH})$, 7.20-7.22(t, 1H, ArH), 7.33-7.36(t, 1H, ArH), 8.05-8.13(d, $J=29.44 \mathrm{~Hz}, 1 \mathrm{H}, \mathrm{NH}) \cdot{ }^{13} \mathrm{C} \mathrm{NMR}\left(100 \mathrm{MHz}, \mathrm{CDCl}_{3}\right)$ : $\delta \mathrm{ppm} \quad 25.25\left(\mathrm{CH}_{2}\right), \quad 26.19\left(\mathrm{CH}_{2}\right), \quad 34.2\left(\mathrm{CH}_{2}\right), \quad 70.52(\mathrm{CH})$, 85.58 $(\mathrm{CH}), 108.7(\mathrm{CH}), 113.27(\mathrm{CH}), 114.1(\mathrm{CH}), 116.43(\mathrm{CH})$, 127.64(CH), 129.9(C), 136.68(C), 154.35(C), 157.18 (C), 160.07(C), 165.35(C), 169.36(C). MS: $m / z=390[\mathrm{M}]^{+}$; Anal. Calcd for $\mathrm{C}_{19} \mathrm{H}_{16} \mathrm{~F}_{2} \mathrm{~N}_{2} \mathrm{O}_{3} \mathrm{~S}$ : C, 58.45; H, 4.13; N, 7.18. Found: C, 58.02; H, 4.03; N, 7.10\%.

4.2.4. N-(2-(3-Chlorophenyl)-4-oxothiazolidine-3-yl)-6-fluorochroman-2-carboxamide (5d). Yield 77\%, mp 151-153 ${ }^{\circ} \mathrm{C}$; IR (DRS): 3401(Amide-NH str.), 3200(Ar, C-H str.), 1717(C=O str.), 1645(amide $\mathrm{C}=\mathrm{O}$ str.), 1550( $-\mathrm{NH}$ bend), 1245(C-F str.), 1201(C-O-C str.), 820(C-Cl str.) $\mathrm{cm}^{-1} ;{ }^{1} \mathrm{H}$ NMR $(400 \mathrm{MHz}$, DMSO): $\delta$ ppm $2.15-2.40(\mathrm{~m}, 2 \mathrm{H}, 2 \mathrm{CH}), 2.75-2.85(\mathrm{~m}, 2 \mathrm{H}$, $2 \mathrm{CH}), 4.66-4.69(\mathrm{~d}, J=16.0 \mathrm{~Hz}, 1 \mathrm{H}, \mathrm{CH}), 3.91-3.95(\mathrm{~d}, J=$ $15.6 \mathrm{~Hz}, 1 \mathrm{H}, \mathrm{CH}), 4.69-4.72(\mathrm{dd}, J=3.6 \mathrm{~Hz}, 3.2 \mathrm{~Hz}, 1 \mathrm{H}, \mathrm{CH})$, 5.92(s, 1H, CH), 6.72-6.95(m, 3H, ArH), 7.24-7.43(m, 4H, $\mathrm{ArH}), 8.0(\mathrm{~s}, 1 \mathrm{H}, \mathrm{NH}) .{ }^{13} \mathrm{C} \mathrm{NMR}\left(100 \mathrm{MHz}, \mathrm{CDCl}_{3}\right): \delta \mathrm{ppm}$ 25.25 $\left(\mathrm{CH}_{2}\right), 26.19\left(\mathrm{CH}_{2}\right), 34.2\left(\mathrm{CH}_{2}\right), 71.78(\mathrm{CH}), 85.58(\mathrm{CH})$, 108.7(CH), 114.1(CH), 116.43(CH), 124.5(CH), 124.93(CH), 126.41(CH), 128(CH), 132.25(C), 140.45(C), 145.35(C), 157.18 (C), 165.35(C), 169.36(C). MS: $m / z=406[\mathrm{M}]^{+}$; Anal. Calcd for $\mathrm{C}_{19} \mathrm{H}_{16} \mathrm{ClFN}_{2} \mathrm{O}_{3} \mathrm{~S}$ : C, 56.09; $\mathrm{H}, 3.96 ; \mathrm{N}, 6.89$. Found: $\mathrm{C}$, $55.90 ; \mathrm{H}, 3.83$; N, $6.83 \%$.
4.2.5. N-(2-(4-Bromophenyl)-4-oxothiazolidine-3-yl)-6-fluorochroman-2-carboxamide (5e). Yield 90\%, mp 118-120 C; IR (DRS): 3452(Amide-NH str.), 3000(Ar, C-H str.), 1725(C=O str.), 1640(amide $\mathrm{C}=\mathrm{O}$ str.), 1545( $-\mathrm{NH}$ bend), 1345(C-F str.), 1251(C-O-C str.), 520(C-Br str.) $\mathrm{cm}^{-1}$; ${ }^{1} \mathrm{H}$ NMR $(400 \mathrm{MHz}$, DMSO- $\left.\mathrm{d}_{6}\right): \delta$ ppm $2.15-2.40(\mathrm{~m}, 2 \mathrm{H}, 2 \mathrm{CH}), 2.75-2.85(\mathrm{~m}, 2 \mathrm{H}$, $2 \mathrm{CH}), 4.63-4.66(\mathrm{~d}, J=16.0 \mathrm{~Hz}, 1 \mathrm{H}, \mathrm{CH}), 3.91-3.95(\mathrm{~d}, J=$ $15.6 \mathrm{~Hz}, 1 \mathrm{H}, \mathrm{CH}), 4.63-4.66(\mathrm{dd}, J=3.6 \mathrm{~Hz}, 3.2 \mathrm{~Hz}, 1 \mathrm{H}$, $\mathrm{CH}), 5.92(\mathrm{~s}, 1 \mathrm{H}, \mathrm{CH}), 6.72-6.95(\mathrm{~m}, 3 \mathrm{H}, \mathrm{ArH}), 7.12-7.85(\mathrm{~m}$, $4 \mathrm{H}, \mathrm{ArH}), 8.0(\mathrm{~s}, 1 \mathrm{H}, \mathrm{NH}) .{ }^{13} \mathrm{C}$ NMR $(100 \mathrm{MHz}, \mathrm{DMSO}-$ $\left.\mathrm{d}_{6}\right): \delta$ ppm $25.25\left(\mathrm{CH}_{2}\right), 26.19\left(\mathrm{CH}_{2}\right), 34.2\left(\mathrm{CH}_{2}\right), 70.50(\mathrm{CH})$, 85.58(CH), 108.7(CH), 114.1(CH), $116.43(\mathrm{CH}), 120.41(\mathrm{C})$, 126.7(CH), 129.36(CH), 129.9(C), 138.93(C), 154.35(C), 157.18 (C), 165.35(C), 169.36(C). MS: $m / z=452[\mathrm{M}+1]^{+}$; Anal. Calcd for $\mathrm{C}_{19} \mathrm{H}_{16} \mathrm{BrFN}_{2} \mathrm{O}_{3} \mathrm{~S}$ : C, 50.56; H, 3.57; N, 6.21. Found: C, $50.45 ; \mathrm{H}, 3.28 ; \mathrm{N}, 6.11 \%$.

4.2.6. 6-Fluoro-N-(2-(4-hydroxyphenyl)-4-oxothiazolidine-3yl) chroman-2-carboxamide (5f). Yield 73\%, mp $132-134^{\circ} \mathrm{C}$; IR (DRS): 3452(Amide-NH str.), (-OH, broad), 3011(Ar, C-H str.), 1728 (C=O str.), 1645(amide $\mathrm{C}=\mathrm{O}$ str.), 1545($\mathrm{NH}$ bend), 1352 (C-F str.), $1278(\mathrm{C}-\mathrm{O}-\mathrm{C}$ str. $) \mathrm{cm}^{-1} ;{ }^{1} \mathrm{H}$ NMR (400 MHz, DMSO-d 6 ): $\delta$ ppm 2.15-2.40(m, 2H, 2CH), $2.75-2.85(\mathrm{~m}, 2 \mathrm{H}, 2 \mathrm{CH}), 4.63-4.66(\mathrm{~d}, J=16.0 \mathrm{~Hz}$, $1 \mathrm{H}, \mathrm{CH}), 3.91-3.95(\mathrm{~d}, J=15.6 \mathrm{~Hz}, 1 \mathrm{H}, \mathrm{CH}), 4.63-$ $4.66(\mathrm{dd}, J=3.6 \mathrm{~Hz}, 3.2 \mathrm{~Hz}, 1 \mathrm{H}, \mathrm{CH}), 5.35(\mathrm{~s}, 1 \mathrm{H}$, $\mathrm{OH}), 5.92(\mathrm{~s}, 1 \mathrm{H}, \mathrm{CH}), 6.72-6.95(\mathrm{~m}, 3 \mathrm{H}, \mathrm{ArH}), 7.14-7.86(\mathrm{~m}$, $4 \mathrm{H}, \mathrm{ArH}), 8.0(\mathrm{~s}, 1 \mathrm{H}, \mathrm{NH}) .{ }^{13} \mathrm{C} \mathrm{NMR}(100 \mathrm{MHz}, \mathrm{DMSO}-$ $\left.\mathrm{d}_{6}\right): \delta$ ppm $25.25\left(\mathrm{CH}_{2}\right), 26.19\left(\mathrm{CH}_{2}\right), 34.2\left(\mathrm{CH}_{2}\right), 55.33\left(\mathrm{CH}_{3}\right)$, $70.50(\mathrm{CH}), 85.58(\mathrm{CH}), 108.7(\mathrm{CH}), 114.1(\mathrm{CH}), 116.43(\mathrm{CH})$, 116.68(C), 125.81(CH), 129.9(C), 132.68(C), 154.35(C), 157.46 (C), 157.18(C), 165.35(C), 169.36(C). MS: $m / z=388[\mathrm{M}]^{+}$; Anal. Calcd for $\mathrm{C}_{19} \mathrm{H}_{17} \mathrm{FN}_{2} \mathrm{O}_{4} \mathrm{~S}$ : C, 58.75; H, 4.41; N, 7.21. Found: C, 58.56; H, 4.34; N, 7.06\%.

4.2.7. 6-Fluoro-N-(4-oxo-2-(p-tolyl) thiazolidin-3-yl) chroman-2-carboxamide (5g). Yield 84\%, mp 89-91 ${ }^{\circ} \mathrm{C}$; IR (DRS): 3328(Amide-NH str.), 2998(Ar, C-H str.), 2900(C-H str.), 1741( $\mathrm{C}=\mathrm{O}$ str.), 1652(amide $\mathrm{C}=\mathrm{O}$ str.), 1548(-NH bend), 1371(C-F str.), 1300(C-O-C str.) $\mathrm{cm}^{-1} ;{ }^{1} \mathrm{H}$ NMR $(400 \mathrm{MHz}$, DMSO- $\left.\mathrm{d}_{6}\right): \delta$ ppm $2.15-2.32(\mathrm{~m}, 2 \mathrm{H}, 2 \mathrm{CH}), 2.34(\mathrm{~s}, 3 \mathrm{H}, 3 \mathrm{CH})$, 2.75-2.85(m, 2H, 2CH), 4.63-4.66(d, $J=16.0 \mathrm{~Hz}, 1 \mathrm{H}, \mathrm{CH})$, $3.91-3.95(\mathrm{~d}, J=15.6 \mathrm{~Hz}, 1 \mathrm{H}, \mathrm{CH}), 4.63-4.66(\mathrm{dd}, J=$ $3.6 \mathrm{~Hz}, 3.2 \mathrm{~Hz}, 1 \mathrm{H}, \mathrm{CH}), 5.92(\mathrm{~s}, 1 \mathrm{H}, \mathrm{CH}), 6.72-6.95(\mathrm{~m}, 3 \mathrm{H}$, $\mathrm{ArH}), 7.11(\mathrm{~m}, 4 \mathrm{H}, \mathrm{ArH}), 8.0(\mathrm{~s}, 1 \mathrm{H}, \mathrm{NH}) .{ }^{13} \mathrm{C} \mathrm{NMR}(100 \mathrm{MHz}$, DMSO-d $\left.{ }_{6}\right): \delta$ ppm 21.03 $\left(\mathrm{CH}_{3}\right), 25.25\left(\mathrm{CH}_{2}\right), 26.19\left(\mathrm{CH}_{2}\right)$, $34.2\left(\mathrm{CH}_{2}\right), 70.50(\mathrm{CH}), 85.58(\mathrm{CH}), 108.7(\mathrm{CH}), 114.1(\mathrm{CH})$, 116.43(CH), 126.73(CH), 127.98(CH), 129.9(C), 136.48(C), 136.52(C), 154.35(C), 157.18(C), 165.35(C), 169.36(C). MS: $\mathrm{m} / z$ $=386[\mathrm{M}]^{+}$; Anal. Calcd $\mathrm{C}_{20} \mathrm{H}_{19} \mathrm{FN}_{2} \mathrm{O}_{3} \mathrm{~S}: \mathrm{C}, 62.16 ; \mathrm{H}, 4.96 ; \mathrm{N}$, 7.25. Found: $\mathrm{C}, 62.07 ; \mathrm{H}, 4.55 ; \mathrm{N}, 7.09 \%$.

4.2.8. N-(2-(2-Chlorophenyl)-4-oxothiazolidine-3-yl)-6-fluorochroman-2-carboxamide (5h). Yield $71 \%, \mathrm{mp} 158-160^{\circ} \mathrm{C}$; IR (DRS): 3412(Amide-NH str.), 3212(Ar, C-H str.), 1725( $\mathrm{C}=\mathrm{O}$ str.), 1685(amide $\mathrm{C}=\mathrm{O}$ str.), 1558(-NH bend), 1251(C-F str.), 1228(C-O-C str.), 800(C-Cl str.), 770(o-di 
substituted) $\mathrm{cm}^{-1} ;{ }^{1} \mathrm{H}$ NMR (400 MHz, DMSO): $\delta$ ppm 2.15$2.32(\mathrm{~m}, 2 \mathrm{H}, 2 \mathrm{CH}), 2.75-2.85(\mathrm{~m}, 2 \mathrm{H}, 2 \mathrm{CH}), 4.63-4.66(\mathrm{~d}$, $J=16.0 \mathrm{~Hz}, 1 \mathrm{H}, \mathrm{CH}), 3.91-3.95(\mathrm{~d}, J=15.6 \mathrm{~Hz}, 1 \mathrm{H}, \mathrm{CH})$, $4.63-4.66(\mathrm{dd}, J=3.6 \mathrm{~Hz}, 3.2 \mathrm{~Hz}, 1 \mathrm{H}, \mathrm{CH}), 5.92(\mathrm{~s}, 1 \mathrm{H}, \mathrm{CH})$, 6.72-6.95(m, 3H, ArH), 7.17-7.65(m, 4H, ArH), 8.0(s, 1H, $\mathrm{NH}) .{ }^{13} \mathrm{C} \mathrm{NMR}\left(100 \mathrm{MHz}, \mathrm{CDCl}_{3}\right): \delta \mathrm{ppm} \quad 25.25\left(\mathrm{CH}_{2}\right)$, 26.19 $\left(\mathrm{CH}_{2}\right), 34.2\left(\mathrm{CH}_{2}\right), 67.50(\mathrm{CH}), 85.58(\mathrm{CH}), 108.7(\mathrm{CH})$, $114.1(\mathrm{CH}), 116.43(\mathrm{CH}), 125.18(\mathrm{CH}), 125.89(\mathrm{CH}), 127.02(\mathrm{CH})$, 127.26(CH), 129.9(C), 131.68(C), 133.39(C), 154.35(C), 157.18 (C), 165.35(C), 169.36(C). MS: $m / z=406[\mathrm{M}]^{+}$; Anal. Calcd for $\mathrm{C}_{19} \mathrm{H}_{16} \mathrm{ClFN}_{2} \mathrm{O}_{3} \mathrm{~S}$ : C, 56.09; H, 3.96; N, 6.89. Found: $\mathrm{C}$, 55.93; H, 3.77; N, 6.84\%.

4.2.9. 6-Fluoro-N-(2-(4-nitrophenyl)-4-oxothiazolidine-3-yl) chroman-2-carboxamide (5i). Yield 79\%, mp 207-209 ${ }^{\circ} \mathrm{C}$; IR (DRS): 3398(Amide-NH str.), 3212(Ar, C-H str.), 1728(C=O str.), 1688(amide $\mathrm{C}=\mathrm{O}$ str.), 1543( $-\mathrm{NH}$ bend), 1595( $\mathrm{N}=\mathrm{O}$ str.), 1272(C-O-C str.), 1290(C-F str.) 790(p-di substituted) $\mathrm{cm}^{-1}$; ${ }^{1} \mathrm{H}$ NMR $\left(400 \mathrm{MHz}, \mathrm{CDCl}_{3}\right): \delta \mathrm{ppm} 2.15-2.32(\mathrm{~m}, 2 \mathrm{H}, 2 \mathrm{CH})$, $2.75-2.85(\mathrm{~m}, 2 \mathrm{H}, 2 \mathrm{CH}), 4.63-4.66(\mathrm{~d}, J=16.0 \mathrm{~Hz}, 1 \mathrm{H}$, $\mathrm{CH}), 3.91-3.95(\mathrm{~d}, J=15.6 \mathrm{~Hz}, 1 \mathrm{H}, \mathrm{CH}), 4.63-4.66(\mathrm{dd}$, $J=3.6 \mathrm{~Hz}, 3.2 \mathrm{~Hz}, 1 \mathrm{H}, \mathrm{CH}), 5.92(\mathrm{~s}, 1 \mathrm{H}, \mathrm{CH}), 6.72-6.95(\mathrm{~m}$, $3 \mathrm{H}, \mathrm{ArH}), 7.49-8.14(\mathrm{~m}, 4 \mathrm{H}, \mathrm{ArH}), 8.0(\mathrm{~s}, 1 \mathrm{H}, \mathrm{NH}) .{ }^{13} \mathrm{C}$ $\mathrm{NMR}\left(100 \mathrm{MHz}, \mathrm{CDCl}_{3}\right): \delta \mathrm{ppm} 25.25\left(\mathrm{CH}_{2}\right), 26.19\left(\mathrm{CH}_{2}\right)$, $34.2\left(\mathrm{CH}_{2}\right), 70.52(\mathrm{CH}), 85.58(\mathrm{CH}), 108.7(\mathrm{CH}), 114.1(\mathrm{CH})$, $116.43(\mathrm{CH}), 122.91(\mathrm{CH}), 126.32(\mathrm{CH}), 129.9(\mathrm{C}), 144.08(\mathrm{C})$, 145.35(C), 154.35 (C), 157.18(C), 165.35(C), 169.36(C). MS: $m / z$ $=417[\mathrm{M}]^{+}$; Anal. Calcd for $\mathrm{C}_{17} \mathrm{H}_{14} \mathrm{FN}_{3} \mathrm{O}_{2}$ : C, 54.67; H, 3.86; N, 10.07. Found: C, 54.39; H, 3.79; N, 9.89\%.

4.2.10. N-(2-(3-Bromophenyl)-4-oxothiazolidine-3-yl)-6-fluorochroman-2-carboxamide (5j). Yield $88 \%$, mp $166-168^{\circ} \mathrm{C}$; IR (DRS): 3482(Amide-NH str.), 3110(Ar, C-H str.), 1745(C=O str.), 1590 (amide $\mathrm{C}=\mathrm{O}$ str.), $1485(-\mathrm{NH}$ bend), $1354(\mathrm{C}-\mathrm{F}$ str.), 1281 (C-O-C str.), 680 (m-di substituted), $520(\mathrm{C}-\mathrm{Br}$ str.) $\mathrm{cm}^{-1} ;{ }^{1} \mathrm{H}$ NMR $\left(400 \mathrm{MHz}, \mathrm{CDCl}_{3}\right): \delta$ ppm 2.15-2.32(m, $2 \mathrm{H}, 2 \mathrm{CH}), 2.75-2.85(\mathrm{~m}, 2 \mathrm{H}, 2 \mathrm{CH}), 4.63-4.66(\mathrm{~d}, J=$ $16.0 \mathrm{~Hz}, 1 \mathrm{H}, \mathrm{CH}), 3.91-3.95(\mathrm{~d}, J=15.6 \mathrm{~Hz}, 1 \mathrm{H}, \mathrm{CH})$, $4.63-4.66(\mathrm{dd}, J=3.6 \mathrm{~Hz}, 3.2 \mathrm{~Hz}, 1 \mathrm{H}, \mathrm{CH}), 5.92(\mathrm{~s}, 1 \mathrm{H}$, $\mathrm{CH}), 6.72-6.95(\mathrm{~m}, 3 \mathrm{H}, \mathrm{ArH}), 7.22-7.41(\mathrm{~m}, 4 \mathrm{H}, \mathrm{ArH}), 8.0(\mathrm{~s}$,

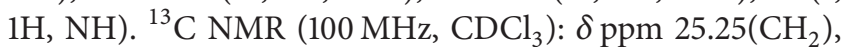
26.19 $\left(\mathrm{CH}_{2}\right), 34.2\left(\mathrm{CH}_{2}\right), 70.32(\mathrm{CH}), 85.58(\mathrm{CH}), 108.7(\mathrm{CH})$, $114.1(\mathrm{CH}), 116.43(\mathrm{CH}), 120.91(\mathrm{C}), 127.55(\mathrm{CH}), 127.95(\mathrm{CH})$, 128.17(CH), 128.74(CH), 129.9(C), 140.65(C), 154.35(C), 157.18 (C), 165.35(C), 169.36(C).MS: $m / z=452[\mathrm{M}+1]^{+}$; Anal. Calcd for $\mathrm{C}_{19} \mathrm{H}_{16} \mathrm{FN}_{3} \mathrm{O}_{5} \mathrm{~S}$ : C, 50.56; H, 3.57; N, 6.21. Found: C, 50.17; $\mathrm{H}, 3.14 ; \mathrm{N}, 6.12 \%$.

\section{Conflict of Interests}

The authors do not have a direct financial relation with the commercial identity mentioned in the paper that might lead to a conflict of interests for any of the authors.

\section{Acknowledgments}

The authors are thankful to the department of chemistry for providing laboratory facilities. The authors are thankful for facilities and grants given under UGC-SAP for Department Research Support (DRS) and Department of Science and Technology (DST) New Delhi for Fund for Improvement of Science and Technology (FIST). The authors are also thankful to RSIC Chandigarh for providing ${ }^{1} \mathrm{H}$ NMR and ${ }^{13} \mathrm{C} N M R$ spectral analysis of the compounds.

\section{References}

[1] I. Kers, G. Csjernyik, I. MacSari et al., "Structure and activity relationship in the (S)-N-chroman-3-ylcarboxamide series of voltage-gated sodium channel blockers," Bioorganic and Medicinal Chemistry Letters, vol. 22, no. 17, pp. 5618-5624, 2012.

[2] T. Nishida, H. Yoshinaga, T. Toyoda, and M. Toshima, "Firstand second-generation practical syntheses of chroman-4-one derivative: a key intermediate for the preparation of SERT/5HT1A dual inhibitors," Organic Process Research and Development, vol. 16, no. 4, pp. 625-634, 2012.

[3] F. Mauler and E. Horváth, "Neuroprotective efficacy of repino$\tan \mathrm{HCl}$, a 5-HT1A receptor agonist, in animal models of stroke and traumatic brain injury," Journal of Cerebral Blood Flow and Metabolism, vol. 25, no. 4, pp. 451-459, 2005.

[4] S. B. Ross, S. Thorberg, E. Jerning et al., "Robalzotan (NAD299), a novel selective 5-HT(1A) receptor antagonist," CNS Drug Reviews, vol. 5, no. 3, pp. 213-232, 1999.

[5] T. J. Cleophas, I. Grabowsky, M. G. Niemeyer, W. N. Mäkel, and E. E. van der Wall, "Long-term efficacy of nebivolol monotherapy in patients with hypertension," Current Therapeutic Research-Clinical and Experimental, vol. 62, no. 6, pp. 451461, 2001.

[6] S. Shelke, G. Mhaske, S. Gadakh, and C. Gill, "Green synthesis and biological evaluation of some novel azoles as antimicrobial agents," Bioorganic \& Medicinal Chemistry Letters, vol. 20, no. 24, pp. 7200-7204, 2010.

[7] K. Sztanke, T. Tuzimski, J. Rzymowska, K. Pasternak, and M. Kandefer-Szerszeń, "Synthesis, determination of the lipophilicity, anticancer and antimicrobial properties of some fused 1,2,4triazole derivatives," European Journal of Medicinal Chemistry, vol. 43, no. 2, pp. 404-419, 2008.

[8] A. Alanine, L. Anselm, L. Steward, S. Thomi, W. Vifian, and M. D. Groaning, "Synthesis and SAR evaluation of 1,2,4-triazoles as A2A receptor antagonists," Bioorganic and Medicinal Chemistry Letters, vol. 14, no. 3, pp. 817-821, 2004.

[9] K. Zamani, K. Faghihi, M. R. Sangi, and J. Zolgharnein, "Synthesis of some new substituted 1,2,4-triazole and 1,3,4thiadiazole and their derivatives," Turkish Journal of Chemistry, vol. 27, no. 1, pp. 119-125, 2003.

[10] N. Raghav and M. Singh, "Acyl hydrazides and triazoles as novel inhibitors of mammali an cathepsin B and cathepsin H," European Journal of Medicinal Chemistry, vol. 77, pp. 231-242, 2014.

[11] S. P. Singh, S. S. Parmar, K. Raman, and V. I. Stenberg, "Chemistry and biological activity of thiazolidinones," Chemical Reviews, vol. 81, no. 2, pp. 175-203, 1981.

[12] C. V. Kavitha, Basappa, S. N. Swamy et al., "Synthesis of new bioactive venlafaxine analogs: Novel thiazolidin-4-ones as antimicrobials," Bioorganic and Medicinal Chemistry, vol. 14, no. 7, pp. 2290-2299, 2006.

[13] A. Kumar, S. Sharma, K. Bajaj et al., "Some new 2,3,6trisubstituted quinazolinones as potent anti-inflammatory, 
analgesic and COX-II inhibitors," Bioorganic and Medicinal Chemistry, vol. 11, no. 23, pp. 5293-5299, 2003.

[14] S. Sharma, T. Singh, R. Mittal, K. K. Saxena, V. K. Srivastava, and A. Kumar, "A study of anti-inflammatory activity of some novel $\alpha$-amino naphthalene and $\beta$-amino naphthalene derivatives," Archiv der Pharmazie, vol. 339, no. 3, pp. 145-152, 2006.

[15] V. Raja Solomon, W. Haq, K. Srivastava, S. K. Puri, and S. B. Katti, "Synthesis and antimalarial activity of side chain modified 4-aminoquinoline derivatives," Journal of Medicinal Chemistry, vol. 50, no. 2, pp. 394-398, 2007.

[16] H. Zhou, S. Wu, S. Zhai et al., "Design, synthesis, cytoselective toxicity, structure-activity relationships, and pharmacophore of thiazolidinone derivatives targeting drug-resistant lung cancer cells," Journal of Medicinal Chemistry, vol. 51, no. 5, pp. 12421251, 2008.

[17] R. B. Pathak, P. T. Chovatia, and H. H. Parekh, "Synthesis, antitubercular and antimicrobial evaluation of 3-(4-chlorophenyl)4-substituted pyrazole derivatives," Bioorganic and Medicinal Chemistry Letters, vol. 22, no. 15, pp. 5129-5133, 2012.

[18] M. L. Barreca, J. Balzarini, A. Chimirri et al., "Design, synthesis, structure - Activity relationships, and molecular modeling studies of 2,3-diaryl-1,3-thiazolidin-4-ones as potent anti-HIV agents," Journal of Medicinal Chemistry, vol. 45, no. 24, pp. 54105413, 2002.

[19] R. S. Harisha, K. M. Hosamani, and R. S. Keri, "Synthesis, invitro microbial and cytotoxic studies of new benzimidazole derivatives," Archiv der Pharmazie, vol. 342, no. 7, pp. 412-419, 2009.

[20] W. Cunico, C. R. B. Gomes, M. D. L. G. Ferreira, L. R. Capri, M. Soares, and S. M. S. V. Wardell, "One-pot synthesis of 2-isopropyl-3-benzyl-1,3-thiazolidin-4-ones and 2-phenyl3-isobutyl-1,3-thiazolidin-4-ones from valine, arenealdehydes and mercaptoacetic acid," Tetrahedron Letters, vol. 48, no. 35, pp. 6217-6220, 2007.

[21] D. R. St. Laurent, Q. Gao, D. Wu, and M. H. Serrano-Wu, "Regioselective synthesis of 3-(heteroaryl)-iminothiazolidin-4ones," Tetrahedron Letters, vol. 45, no. 9, pp. 1907-1910, 2004. 

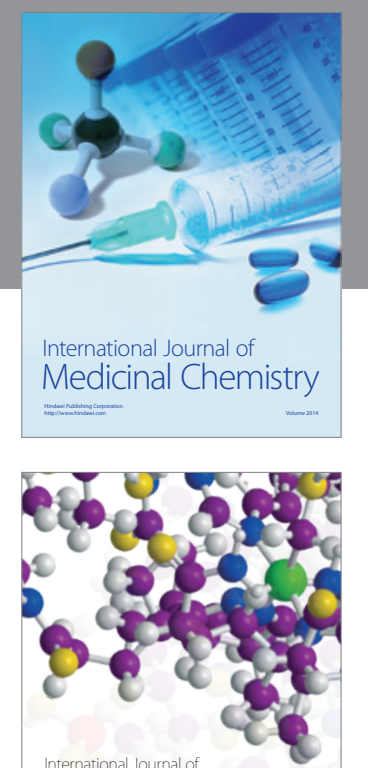

\section{Carbohydrate} Chemistry

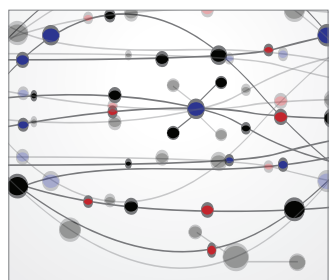

The Scientific World Journal
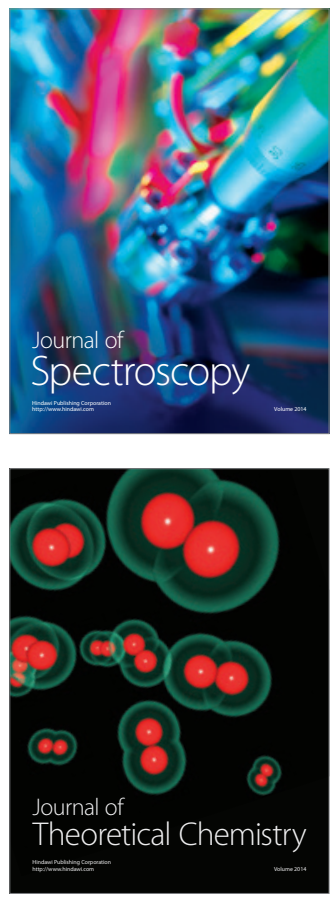
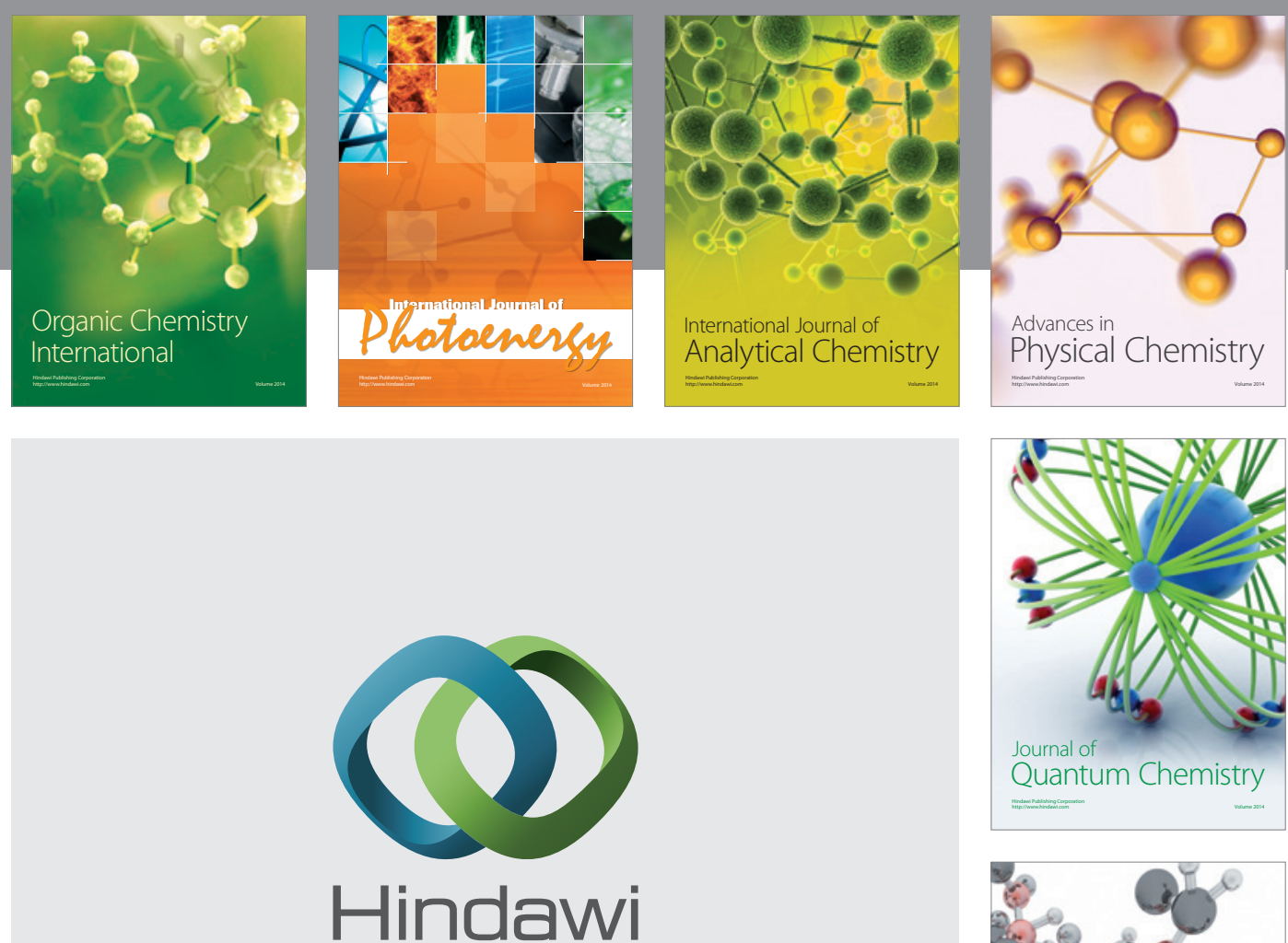

Submit your manuscripts at

http://www.hindawi.com

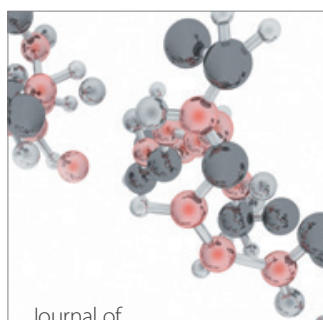

Analytical Methods

in Chemistry

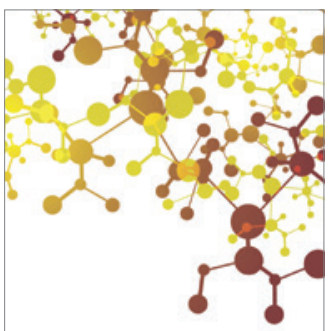

Journal of

Applied Chemistry

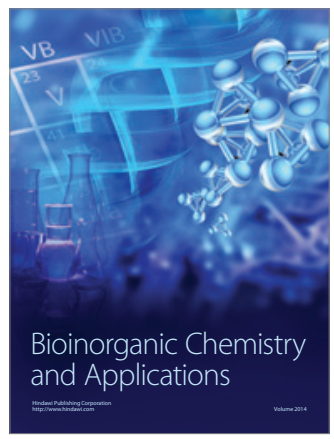

Inorganic Chemistry
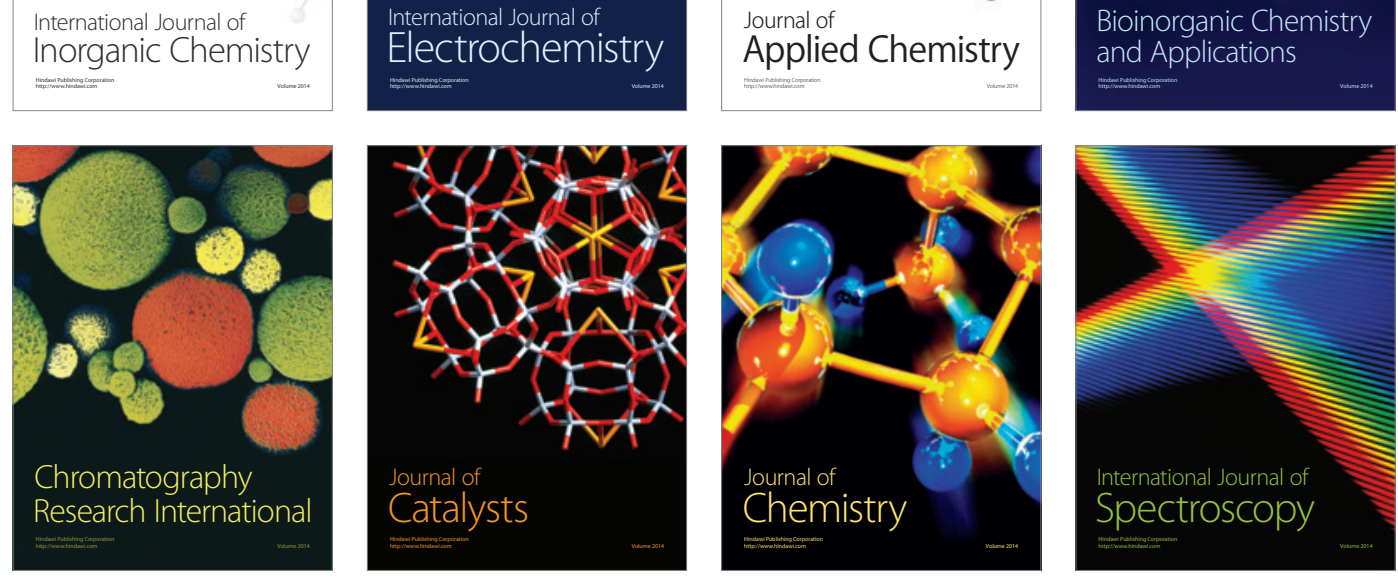